\title{
Measurement of ice-sheet topography using satellite-radar interferometry
}

\author{
IAN Joughin, " Dale WinebrenNer, \\ Applied Physics Laboratory, University of Washington, Seattle, Washington 98105, U.S.A.
}

\author{
MARK FAHNESTOCK, \\ JGESS, Department of Meteorology, University of Maryland at College Park, College Park, Maryland 20742, U.S.A.
}

RON KWOK,

Jet Propulsion Laboratory, California Institute of Technology, Pasadena, Califormia 91109, U.S.A.

WiLLIAM KRABILL

Observational Science Branch, Goddard Space Flight Center, Wallops Flight Facility, Wallops Island, Virginia 23337, U.S.A.

\begin{abstract}
Detailed digital elevation models (DEMs) do not exist for much of the Greenland and Antarctic ice sheets. Radar altimetry is at present the primary, in many cases the only, source of topographic data over the ice sheets, but the horizontal resolution of such data is coarse. Satellite-radar interferometry uses the phase difference between pairs of synthetic aperture radar (SAR) images to measure both ice-sheet topography and surface displacement. We have applied this technique using ERS-1 $\mathrm{SAR}$ data to make detailed (i.e. $80 \mathrm{~m}$ horizontal resolution) maps of surface topography in a $100 \mathrm{~km}$ by $300 \mathrm{~km}$ strip in West Greenland, extending northward from just above Jakobshavns Isbra. Comparison with a $76 \mathrm{~km}$ long line of airborne laser-altimeter data shows that we have achieved a relative accuracy of $2.5 \mathrm{~m}$ along the profile. These observations provide a detailed view of dynamically supported topography near the margin of an ice sheet. In the final section we compare our estimate of topography with phase contours due to motion, and confirm our earlier analysis concerning vertical icesheet motion and complexity in ERS-1 SAR interferograms.
\end{abstract}

\section{INTRODUGTION}

The ice sheets of Greenland and Antarctica play an important role in the Earth's climatic balance. Of particular importance is the possibility of a significant rise in sea level brought on by a change in the mass balance of, or the collapse of, a large ice sheet (Bindschadler, 1991). An understanding of the processes that could lead to such change is hindered by the inability to determine even the current state of the ice sheets. For example, it is not clear whether the mass of the Greenland ice sheet is increasing or decreasing (van der Veen, 1991). Ground-based measurements are capable of sampling only small areas of an ice sheet. Therefore, they may indicate only regional change. Only in the past two decades has satellite remote sensing offered the potential for wide-scale monitoring of ice sheets.

Surface elevation observations are important to many

\footnotetext{
* Present address: Jet Propulsion Laboratory, California Institute of Technology, Pasadena, California 91109, U.S.A
}

aspects of the study of ice sheets. Topographic data are useful for mapping and detecting changes in the boundaries of the individual drainage basins that make up an ice sheet (Bindschadler, 1984). Short-scale (i.e. a few ice thicknesses) undulations in the topography are often caused by obstructions to flow created by the basal topography (Paterson, 1981; Johannesson, 1992). Therefore, surface topography can be used to help infer conditions at the bed (Thomas and others, 1985). Furthermore, densely sampled surface topography is important for modeling glacier dynamics.

Although radar altimeters have been used to measure absolute elevations for ice sheets, they do not have sufficient resolution to measure short-scale topography. Photoclinometry can measure the detailed surface topography of ice sheets Bindschadler and Vornberger, 1994). This method is limited, however, by the need for an extensive, linearly continuous grid of ground-control points. Thus, widespread application of this technique awaits the necessary control data, which must be provided by ground-based or airborne altimetry surveys.

Interferometric synthetic aperture radar (SAR) provides a means to measure both detailed topography 
(Zebker and Goldstein, 1986; Li and Goldstein, 1990) and surface displacement (motion) Gabriel and others, 1989), using the phase difference between pairs of complex SAR images. When the images are acquired at different times from nearly repeating orbits (repeat pass), interferometric phase is affected by both topography and surface displacement. If the images are acquired simultaneously (single pass) with dual SARs, phase is affected only by topography. Thus, repeat-pass interferograms provide motion and topographic data, while single-pass interferograms yield only topographic information. The repeat-pass approach has been applied to spaceborne and airborne SARs, while the single-pass approach has been used only for airborne systems. This paper examines the repeat-pass approach with images from the first European Remote Sensing (ERS-1) SAR.

The topography of ice sheets is characterized by minor undulations with small surface slopes. This type of topography is well suited to interferometric measurement. The Canadian Centre for Remote Sensing (CCRS) airborne SAR has acquired single-pass interferograms to map the topography of glaciers on Bylot Island in the Canadian Arctic Mattar and others, 1994). Motioninduced fringes (modulo- $2 \pi$ phase contours), however, hinder interferometric SAR estimation of topography from repeat-pass (i.e. ERS-1) interferograms. These additional fringes, which may be greater in number than the topographic fringes, make it impossible to estimate topography directly from a single-difference, repeat-pass interferogram. Kwok and others in press) have shown that when the velocity field does not change with time, interferograms can be differenced to cancel icesheet motion and, thus, obtain a topography-only interferogram. This paper examines the use of doubledifference interferograms to measure the detailed topography of ice sheets. Comparison with laser-altimeter data indicates that high-resolution (i.e. $80 \mathrm{~m}$ pixels) digital elevation models (DEMs) with relative accuracies on the order of $2.5 \mathrm{~m}$ can be achieved. These results establish that repeat-pass satellite-radar interferometry can be used to map the topography of ice sheets with an accuracy suitable for many research goals.

This paper begins with a review of interferometry, followed by a description of the double-differencing technique used to cancel the effects of ice-sheet motion Kwok and Fahnestock, 1996). The next section describes the generation of DEMs for a single SAR frame in West Greenland. Airborne laser-altimeter data are used to establish accuracy. Interferometrically derived DEMs from adjacent frames are also examined. Finally, one of the DEMs is compared with the motion-dependent phase variation of an ice-sheet interferogram.

\section{INTERFEROMETRY BAGKGROUND}

\section{Single-difference interferometry}

The geometry of an interferometric SAR is shown in Figure 1. The interferometer acquires two images of the same scene with SARs located at $\mathrm{S}_{1}$ and $\mathrm{S}_{2}$. The first SAR is at altitude $H$. From $\mathrm{S}_{1}$ the range, $r_{0}$, and look angle, $\theta$,

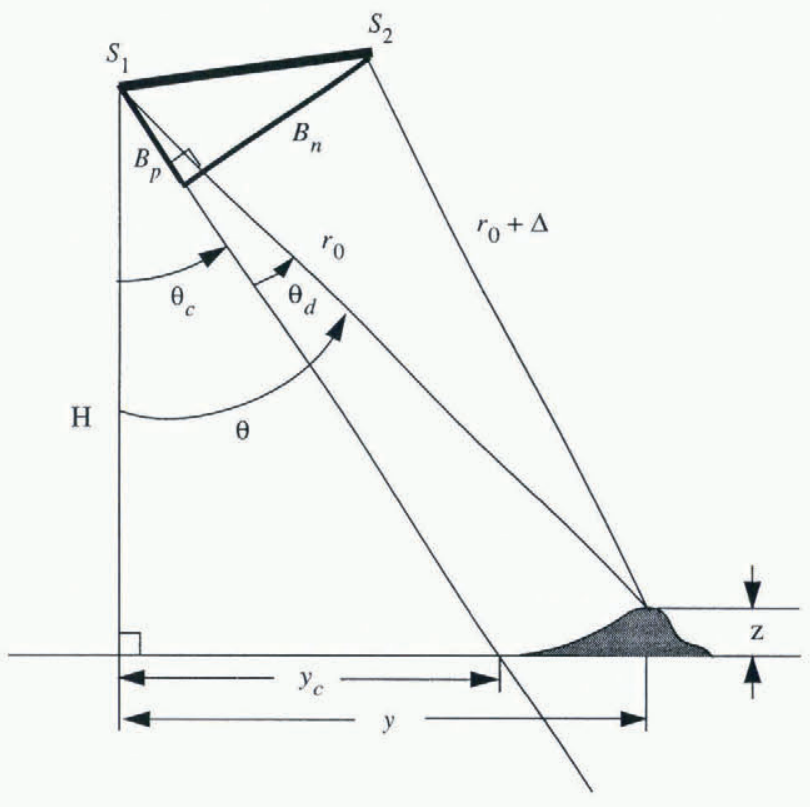

Fig. 1. Geometry of an interferometric SAR.

to a point on the surface are determined by the ground range, $y$, and elevation, $z$. The range to the same point from the $\mathrm{SAR}$ at $\mathrm{S}_{2}$ differs from $r_{0}$ by $\Delta$. For a single-pass system, such as TOPSAR Zebker and others, 1992), two images are acquired simultaneously using separate antennas. In contrast, a repeat-pass interferometer acquires a single image of the same area twice from two nearly repeating orbits or flight lines. The baseline separating the SARs can be expressed in terms of components perpendicular to, $B_{1}$, and parallel to, $B_{\mathrm{p}}$, a reference-look direction. A convenient choice is to let the nominal center-look angle, $\theta_{c}$, define the reference-look direction.

The additional information gained by using an interferometer over a single SAR is the range difference, $\Delta$. The range resolution of a typical SAR is not sufficient to measure this difference accurately for topographic mapping. Instead, the range difference is determined using phase-difference information from the pair of complex SAR images. For a distributed target a pixel in a complex image can be represented as

$$
V_{1}=\exp \left(-j 2 k r_{0}\right) W_{1}=\exp \left(-j 2 k r_{0}\right) A_{1} \mathrm{e}^{j \phi_{1}},
$$

where $k$ is the wave number and $W_{1}$ is a complex, circular Gaussian random variable $(\mathrm{RV})$ with amplitude $A_{1}$ and phase $\phi_{1}$ (Rodriguez and Martin, 1992). Because the modulo- $2 \pi$ phase is uniformly distributed, a single complex image cannot be used to determine range information.

A complex interferogram is formed as the product of one complex SAR image with the complex conjugate of a second. A pixel in the interferogram can be expressed as

$$
V_{1} V_{2}^{*}=\exp \left[-j 2 k r_{0}+j 2 k\left(r_{0}+\Delta\right)\right] W_{1} W_{2}{ }^{*} .
$$

The phase of this product is given by

$$
\angle V_{1} V_{2}{ }^{*}=\left[2 k \Delta+\left(\phi_{1}-\phi_{2}\right)\right]_{\bmod (2 \pi)} .
$$

Although $\phi_{1}$ and $\phi_{2}$ are both uniformly distributed, if $W_{1}$ and $W_{2}$ are correlated their difference, $\left(\phi_{1}-\phi_{2}\right)$, is not 
uniformly distributed Joughin, 1995). In fact, the distribution of the phase difference can be quite sharply peaked if the complex images are well correlated.

Even with a narrow phase distribution, the phase difference is still only known modulo $2 \pi$. A phaseunwrapping algorithm (Goldstein and others, 1988) must be used to remove the modulo- $2 \pi$ ambiguity. The range difference for repeat-pass interferometry can then be determined using

$$
\Delta=\frac{\phi_{\text {unwrap }}}{2 k},
$$

where $\phi_{\text {unwrap }}$ denotes the unwrapped interferometricphase difference.

For a repeat-pass interferometer, $\Delta$ is affected by both topography and any movement of the target between orbits that is directed toward or away from the look direction of the radar. The interferometric phase can be expressed as the sum of motion- and topographydependent terms,

$$
\phi_{\text {unwrap }}=\phi_{\text {topography }}+\phi_{\text {displacement }} .
$$

The displacement-dependent term is given by

$$
\phi_{\text {displacement }}=2 k\left[\left(y_{2}-y_{1}\right) \sin \theta-\left(z_{2}-z_{1}\right) \cos \theta\right] \text {, }
$$

where the subscripts are used to denote the coordinates at the center of the SAR resolution cell during first and second satellite passes. When the effects of motion and topography are separated, $\phi_{\text {displacement }}$ can be used to estimate ice-sheet motion Goldstein and others, 1993; Joughin and others, 1995; Rignot and others, 1995).

In order to examine the effect of the baseline on the interferometric phase, the topography-dependent term can be approximated as

$$
\begin{aligned}
\phi_{\text {topography }} \approx 2 k\left\{\frac{-B_{\mathrm{n}}}{r_{0}}\left[\left(y-y_{\mathrm{c}}\right) \cos \theta_{\mathrm{c}}+z \sin \theta_{\mathrm{c}}\right]\right. \\
\left.-B_{\mathrm{p}} \cos \theta_{\mathrm{d}}+\frac{B^{2}}{2 r_{0}}\right\} .
\end{aligned}
$$

Variation in ground range, $y$, introduces a phase ramp across the interferogram. This ramp can be removed by subtracting the interferogram corresponding to a zeroheight surface. For a fixed range, $r_{0}, y$ and $z$ are not independent variables and are related by

$$
y=\sqrt{r_{0}^{2}-H^{2}+2 z H-z^{2}} .
$$

Applying Equation (8) to compute the phase ramp corresponding to a zero-height surface, the elevationdependent phase variation is given by

$$
\begin{aligned}
& \phi_{z} \approx \frac{-2 k B_{\mathrm{n}}}{r_{0}}[\left(\sqrt{r_{0}^{2}-H^{2}+2 z H-z^{2}}-\sqrt{r_{0}^{2}-H^{2}}\right) \\
&\left.\cdot \cos \theta_{\mathrm{c}}+z \sin \theta_{\mathrm{c}}\right] \\
& \approx \frac{-2 k B_{\mathrm{n}}}{\sin \theta_{\mathrm{c}} r_{0}} z .
\end{aligned}
$$

This indicates that the sensitivity of the interferometer to topography is proportional to the length of $B_{\mathrm{n}}$.

\section{Double differencing to cancel ice-sheet motion}

Motion-dependent phase variation often dominates topography-dependent phase variation in ice-sheet interferograms. The effect of motion must be removed from the interferogram before topography can be estimated. Kwok and others (in press) have shown that the motiondependent phase variation can be cancelled by differencing interferograms. This technique relies on the assumption that ice is displaced by the same amount over the periods spanned by two interferograms. In this case the baseline-independent $\phi_{\text {displacement }}$ terms are identical for each interferogram. If phase ramps are removed from the interferograms, then, applying Equations (5) and (9), these interferograms can be approximated as

and

$$
\phi_{1} \approx 2 k\left(\frac{-B_{\mathrm{n}, 1}}{\sin \theta_{\mathrm{c}} r_{0}} z+\Delta_{\text {displacement }}\right)
$$

$$
\phi_{2} \approx 2 k\left(\frac{-B_{\mathrm{n}, 2}}{\sin \theta_{\mathrm{c}} r_{0}} z+\Delta_{\text {displacement }}\right) .
$$

The two interferograms are differenced to form a doubledifference interferogram,

$$
\phi_{1}-\phi_{2} \approx \frac{-2 k\left(B_{\mathrm{n}, 1}-B_{\mathrm{n}, 2}\right)}{\sin \theta_{c} r_{0}} z .
$$

This result is equivalent to a topography-only interferogram with a baseline equal to the difference of the baselines for the single-difference interferograms .

Figure 2 shows an example of double differencing to cancel ice-sheet motion for interferograms from frame 2169 (see Figure 3 for location). Both single-difference interferograms are formed using $6 \mathrm{~d}$ pairs. These interferograms have not been unwrapped, so the fringes due to the modulo- $2 \pi$ ambiguity are still present. The interferometric pair shown in Figure $2 \mathrm{a}$ was acquired during orbits 3218 and 3304 and has $B_{\mathrm{n}} \approx 2 \mathrm{~m}$. The baseline is small, so this is essentially a motion-only interferogram. Figure $2 \mathrm{~b}$ shows the interferogram for orbits 3132 and 3218 , which has $B_{\mathrm{n}} \approx-183 \mathrm{~m}$. Phase ramps corresponding to zero-height surfaces have been removed from both interferograms. The double-difference interferogram is shown in Figure 2c. The motion-dependent fringes, which are the cause of most of the complexity seen in the singledifference interferograms, are cancelled, leaving only fringes due to topography. The baseline of this topography-only interferogram is approximately $185 \mathrm{~m}$.

Although the interferograms in this example have the same temporal baseline, it is not necessary that both interferograms span the same number of days to cancel motion. For example, the phase of a $3 \mathrm{~d}$ interferogram can be doubled. This doubles the motion-induced phase variation so that the result can be differenced with a $6 \mathrm{~d}$ pair. The baseline of a doubled interferogram is also effectively doubled.

An interferometric pair of complex images must be correlated to succeed in creating a single-difference interferogram. To form a double-difference interferogram, however, the constituent single-difference interferograms do not need to be correlated with each other, because much of the phase variation from speckle is 


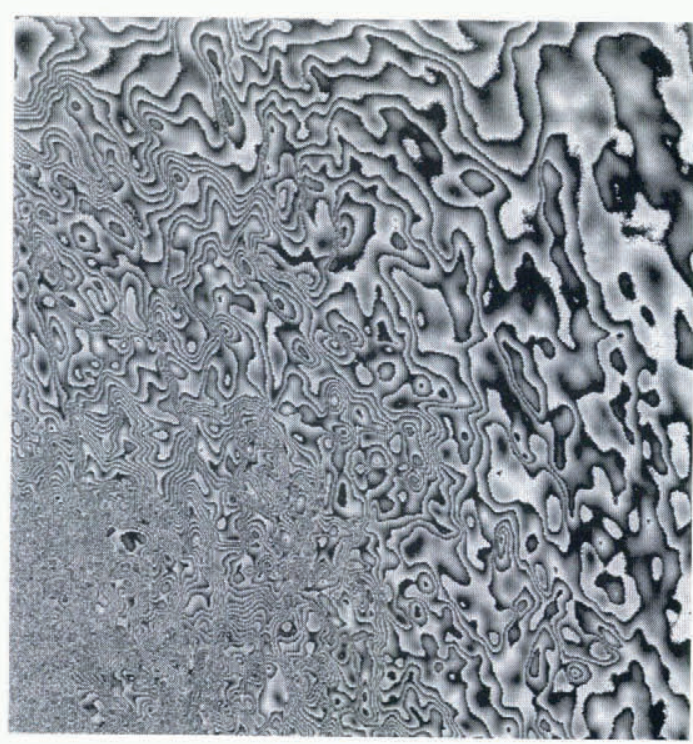

a

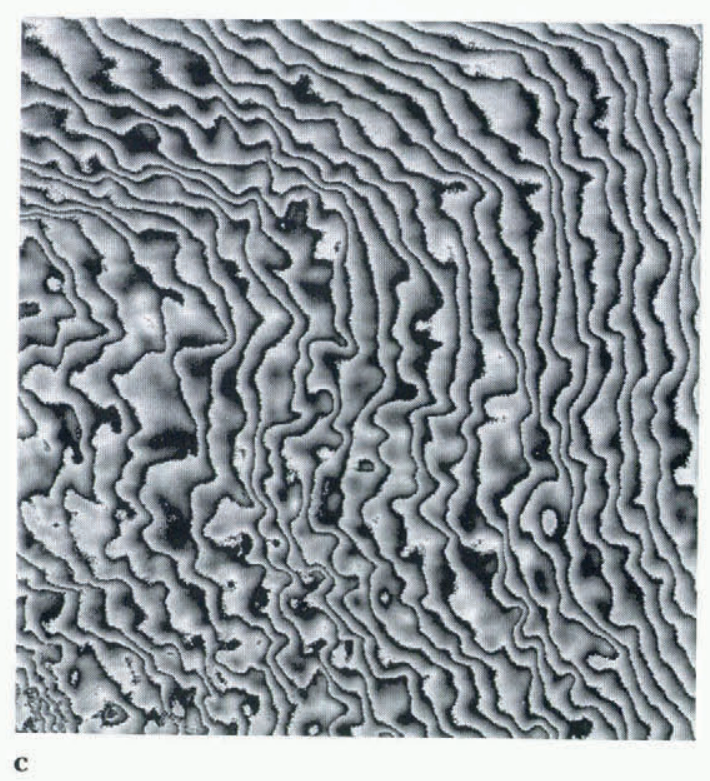

cancelled when the single-difference interferograms are formed. This means that as long as the motion field does not change, interferograms can be differenced even when separated by long intervals. Nevertheless, correlation between interferograms aids in their registration. When interferograms are correlated and the baseline is known, they can be registered with sub-pixel accuracy using the cross-correlation function computed for one image from each interferometric pair (Joughin, 1995).

Where there is no correlation between interferograms the locations of the corner points computed by the SAR processor at the United Kingdom Processing and Archiving Facility (UK-PAF) are used to register the interferograms (Joughin, 1995). The accuracy of this method was determined by comparison with the more accurate cross-correlation method for several interferograms that could be registered with both methods. The interferograms used for this test are separated by intervals of 3,6 and $15 \mathrm{~d}$. Errors for the corner-point registration method are estimated to be $7.5 \mathrm{~m}$ in range and $22.4 \mathrm{~m}$ in azimuth. These are relative errors. For comparison, the nominal absolute accuracy of the corner-point locations is $50 \mathrm{~m}$ (Smith and others, 1994)

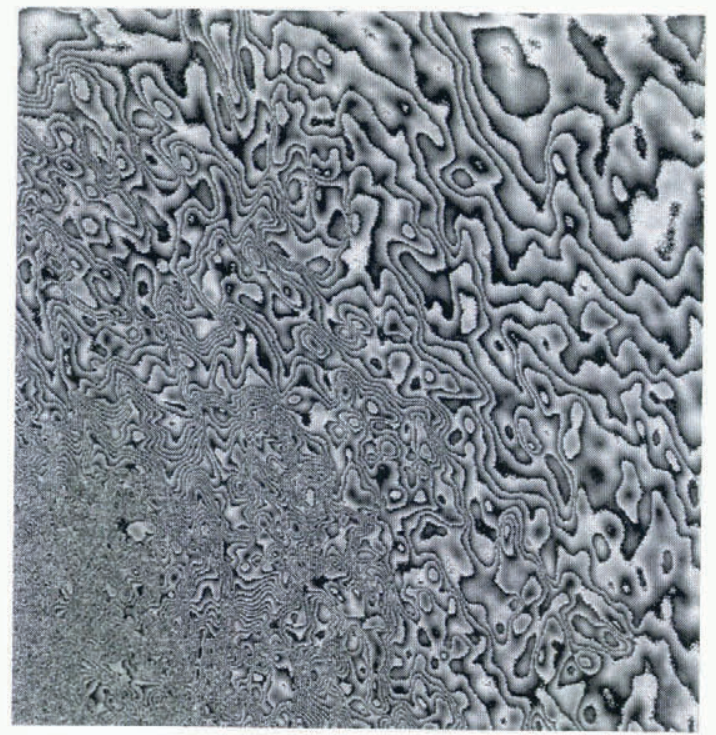

b Fig. 2. An example of double differencing single-difference
interferograms from (a) orbits $3218 / 3304$ and $(b)$ orbits
3132/3218 10 generale (c) a lopography-only interferogram.

\section{DEM GENERATION}

After the double-difference interferograms are formed, the next step is to remove the modulo- $2 \pi$ ambiguity in the phase. This is done with a phase-unwrapping algorithm Goldstein and others, 1988; Joughin, 1995). Because integration of the differenced phase is used to remove the modulo- $2 \pi$ ambiguity, there is always an unknown constant associated with the unwrapped solution, and thus only the relative phase is known. This unknown constant is solved for as part of the baseline estimation procedure (Joughin, 1995).

ERS-1 orbits are not known well enough to estimate baselines with the level of accuracy needed to generate DEMs. As a result, the baseline must be determined using

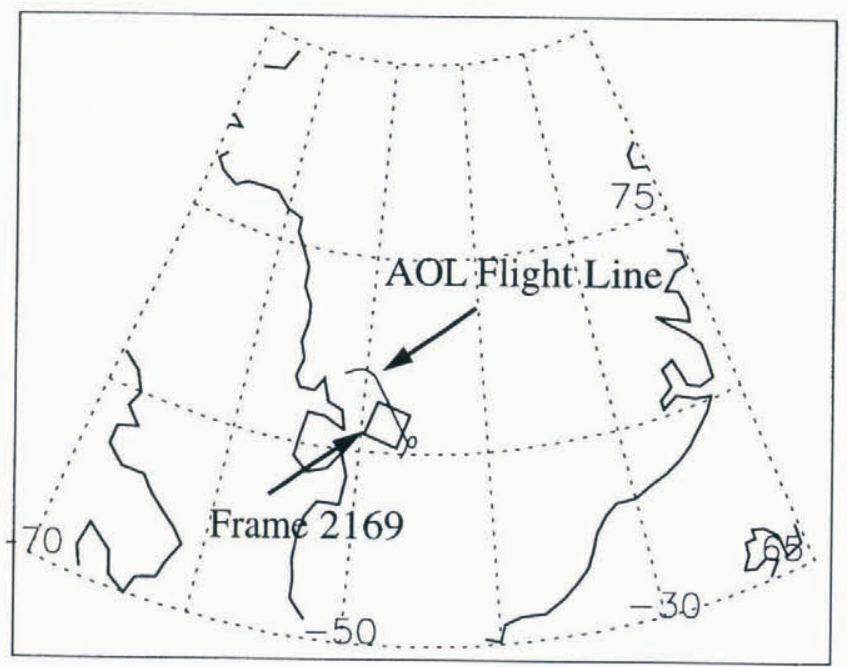

Fig. 3. Localion of frame 2169 and part of the AOL flight line from 27 July 1993. 
tie points (points of known elevation; Zebker and others, 1994a). When the baseline is modelled as varying linearly along track, at least four tie points are needed. A greater number of tie points can be used in the least-squares solution to help reduce the effect of tie-point error on the baseline estimate (Zebker and others, 1994a).

Many of the existing topographic data for ice sheets were acquired using radar-altimetry data, which has coarse horizontal resolution (i.e. a few kilometers). To determine the baseline length, we use tie points extracted from the National Survey and Cadastre of Denmark (KMS) DEM (personal communication from S. Ekholm, 1994), which was derived principally from radar altimetry. Because the ice sheets are relatively smooth, this DEM yields tie points with errors of about $20 \mathrm{~m}$ in the presence of undulating topography. Simulations indicate that baselines estimated using 100 or more tie points extracted from the KMS DEM yield relative errors of about $2-3 \mathrm{~m}$ in the estimated topography (Joughin, 1995).

After baseline estimation, the next step in creating a DEM is to add back the phase ramps that were removed prior to phase unwrapping. The range difference is then estimated by scaling the unwrapped phase using the relation given by Equation (4). The baseline and $\Delta$ are used to solve for the look angle, $\theta$ (Li and Goldstein, 1990; Rodriguez and Martin, 1992). Elevation and ground range, relative to a curved earth, are computed using (Joughin, 1995)

$z=-R_{\mathrm{e}}+\sqrt{R_{\mathrm{e}}{ }^{2}-2\left(R_{\mathrm{e}}+H\right) r_{0} \cos \theta+r_{0}{ }^{2}+2 H R_{\mathrm{e}}+H^{2}}$

and

$y=R_{\mathrm{e}} \arccos \left(\frac{2 H R_{\mathrm{e}}+H^{2}+z^{2}-r_{0}^{2}+2 R_{\mathrm{e}}\left(R_{\mathrm{e}}+z\right.}{2\left(R_{\mathrm{e}}+z\right)\left(R_{\mathrm{e}}+H\right)}\right)$

The final step in DEM generation is to interpolate the unevenly spaced ground-range and elevation values to an evenly spaced grid.

\section{INTERFEROMETRICALLY DERIVED DEMS FOR AN AREA IN WEST GREENLAND}

\section{Study area and interferograms}

This section examines DEMs created for the area covered by frame 2169 from track 25 of the ERS-1 SAR during the first and second ice phases. The location of this approximately $100 \mathrm{~km}$ by $100 \mathrm{~km}$ frame is shown in Figure 3. Four single-difference interferograms were generated using data from six orbits. Table 1 gives the orbit pairs, temporal baselines (time between the acquisition of images in an interferometric pair) and ESA baseline estimates (Solaas, 1994) for the single-difference interferograms. Each interferogram in the table is assigned a number, $n$, that is used for subsequent identification. Interferograms are then denoted as $I_{n}$. These interferograms are created using ERS-1, full-scene, complex images processed at the UK-PAF. The complex interferograms are multilook-averaged 4 pixels in range by 20 pixels in azimuth to yield a pixel spacing of approximately $80 \mathrm{~m}$. As part of the processing, phase ramps corresponding to a zero-height surface are removed.

The interferograms listed in Table 1 are used to create six double-difference interferograms, which are identified in
Table 1. Orbit numbers and ESA baseline estimates for interferograms generated for frame 2169

\begin{tabular}{crrrrr}
\hline $\begin{array}{c}\text { Interfero- } \\
\text { gram }\end{array}$ & $\begin{array}{r}\text { First } \\
\text { orbil }\end{array}$ & $\begin{array}{c}\text { Second } \\
\text { orbit }\end{array}$ & $\Delta T$ & \multicolumn{1}{c}{$B_{\mathrm{n}}$} & \multicolumn{1}{c}{$B_{\mathrm{p}}$} \\
& & & $\mathrm{d}$ & $\mathrm{m}$ & $\mathrm{m}$ \\
\hline$I_{1}$ & 12838 & 12881 & 3 & 165.75 & 73.25 \\
$I_{2}$ & 3089 & 3132 & 3 & 59.71 & 30.70 \\
$I_{3}$ & 3132 & 3218 & 6 & -182.70 & -4.96 \\
$I_{4}$ & 3218 & 3304 & 6 & 1.56 & 23.00 \\
\hline
\end{tabular}

Table 2. The sequence of operations and the singledifference interferograms used to form each doubledifference result are included in the far lefthand column of Table 2. The baselines in this table are derived from the baseline estimates given in Table 1. Three of the doubledifference interferograms do not include $I_{1}$. For these interferograms registration is performed by cross-correlating one image from each pair after compensation for the baseline. Because they are separated by 22 months, there is no coherence between $I_{1}$ and the other interferograms. As a result, double-differenced pairs that include $I_{1}$ are registered using the corner-point registration algorithm mentioned earlier. The complex interferograms are doubledifferenced by multiplying one interferogram with the complex conjugate of the other. Phase doubling of a $3 \mathrm{~d}$ complex interferogram to match the motion of a $6 \mathrm{~d}$ interferogram, denoted as $2 \times I_{n}$, is achieved by multiplying it with itself. To estimate the baseline, 132 evenly spaced tie points, $z_{t}$, were extracted from the KMS DEM (personal communication from S. Ekholm, 1994).

DEMs generated directly from the double-difference interferograms have large errors (i.e. a few hundred meters) (Joughin, 1995), which vary primarily in the azimuth direction. The length scale of these errors is greater than about $15 \mathrm{~km}$. It is likely that these errors are the result of phase irregularities in the complex images from the UKPAF. We estimate the error by low-pass filtering the difference between the interferogram and a synthetic interferogram created using the KMS DEM. Interfero-

Table 2. Double-difference interferograms for frame 2169 and corresponding baselines derived from ESA baselines

Double-difference
interferogram $\quad B_{\mathrm{n}} \quad B_{\mathrm{p}}$

$\mathrm{m}$

$\mathrm{m}$

$\begin{array}{crr}I_{2}-I_{1} & -106.04 & 42.55 \\ 2 \times I_{2}-I_{3} & 302.12 & 66.36 \\ 2 \times I_{2}-I_{4} & 117.86 & 84.40 \\ 2 \times I_{1}-I_{3} & 514.20 & 151.46 \\ I_{4}-I_{3} & 184.26 & -18.04 \\ 2 \times I_{1}-I_{4} & 329.94 & 169.5\end{array}$


grams are corrected by subtracting this estimate of the error (Joughin, 1995). In the resulting DEM the kilometer-scale information is provided by the interferogram, while the longer-scale information (greater than $16 \mathrm{~km}$ ) is supplied by the K.MS DEM. This is not the ideal solution, however, as it allows long-wavelength errors in the altimetry-derived KMS DEM to be propagated to the interferometrically derived DEM. Further research is needed to establish the cause of the underlying long-wavelength phase errors in the interferograms so that ultimately they can be eliminated without the aid of a supplementary DEM. For now, this correction allows us to proceed with our investigation of interferometric estimation of ice-sheet topography.

\section{Analysis of interferometrically derived DEMs}

After correction for long-wavelength errors, the interferograms are used to create a set of six DEMs. These DEMs are averaged together to create a composite DEM, $z_{\mathrm{i}}$. This
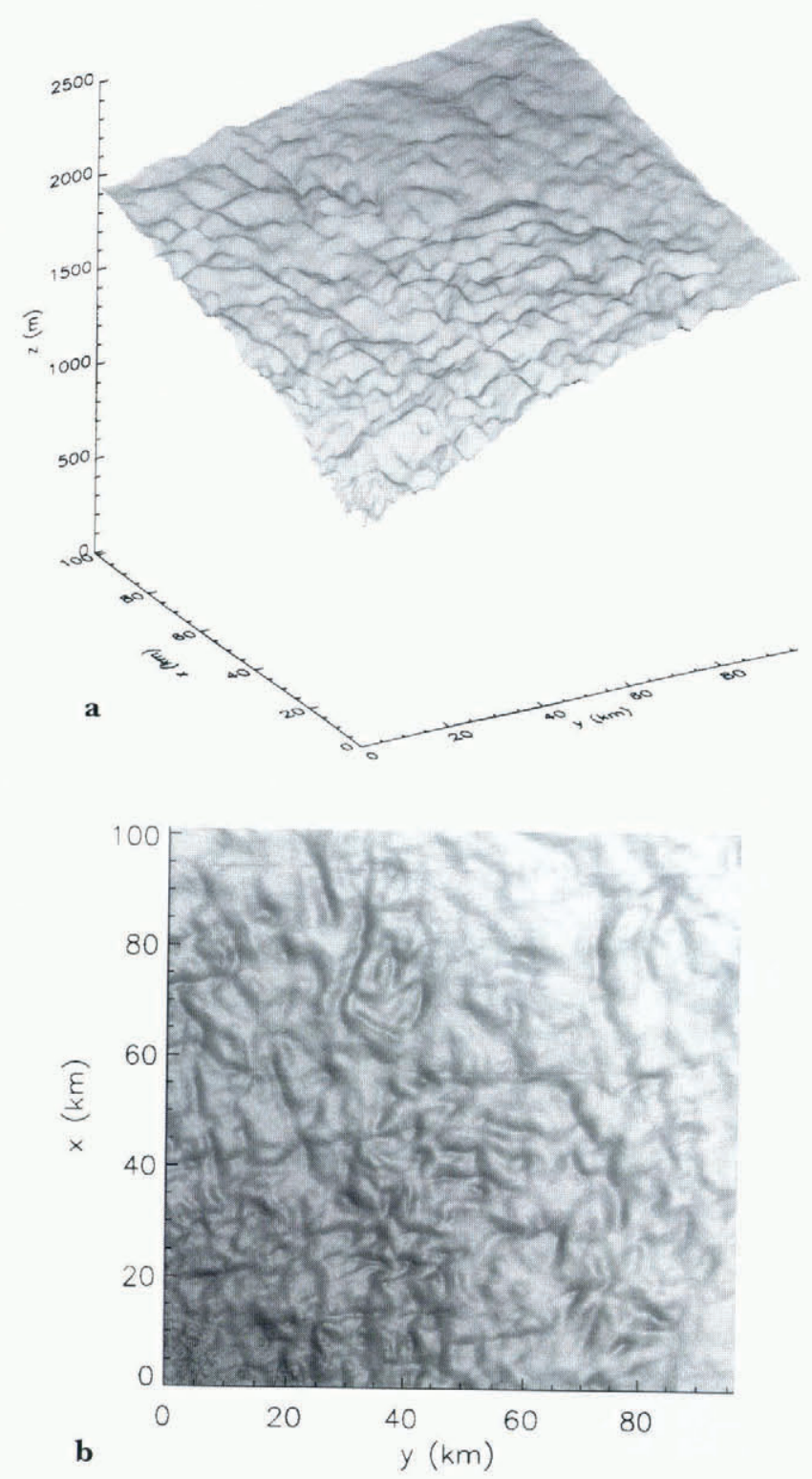

Fig. 4. Shaded surface of the composile DEM for frame 2169. The vantage-point is (a) from the side and (b) from above. The light source is directed from above, along the $z$ axis.
DEM is displayed in Figure $4 \mathrm{a}$ as a shaded surface with an illumination source directed from above, along the $z$ axis. The topography consists of a gentle slope extending from about $2400 \mathrm{~m}$ in the top righthand corner to about $1100 \mathrm{~m}$ in the lower lefthand corner. Kilometer-scale undulations typical of ice-sheet terrain are clearly visible. To better illustrate these features, Figure $4 \mathrm{~b}$ shows the DEM as a shaded surface from a vantage-point directly overhead. The light source is the same as that used in Figure 4a. Visually, this enhances the appearance of short-scale undulations, while de-emphasizing the long-scale trend of the slope.

There are no detailed DEMs for the entire SAR frame to compare with the interferometric DEMs. Instead, differences between the individual DEMs and the composite DEM are examined. Because the error for $z_{\text {a }}$ is unknown, these differences are measures of DEM consistency rather than accuracy. Nevertheless, it is instructive to examine how the DEMs differ. Accuracy is evaluated by comparison with laser-altimeter data for a single flight line in the next sub-section.

Mean and standard deviations of the difference, $\hat{z}-z_{\mathrm{a}}$, are given in the second and third columns of Table 3 . The mean difference, $\mu_{\hat{z}-z_{u}}$, varies by about $\pm 3 \mathrm{~m}$. Because mean differences should be determined largely by tie-point bias, which is the same for all the DEMs, the variability in these differences should be smaller than observed.

Standard deviations range from moderate to quite large. In general, smaller values correspond to the interferograms with the larger baselines. The differences are too large to be explained by baseline-estimation error alone (Joughin, 1995). It is also unlikely that random phase error due to speckle can explain these differences, because the interferograms have been heavily multilook-averaged. Localized phase-unwrapping errors could increase the error, but only for those parts of the images where correlation is low. Most of the unwrapping errors are confined to the lower lefthand corner of the frame, where motion is greatest. Elevations are less than $1400 \mathrm{~m}$ in this region of the DEM. Phase-unwrapping errors are eliminated from the comparison by computing the standard deviation only for that part of the DE.M where elevation is greater than $1400 \mathrm{~m}$. These values, reported in the last column of Table 4, do not show a reduction significant enough to attribute the large values of $\sigma_{z-z_{\mathrm{i}}}$ to errors in

Table 3. Comparison of interferometrically derived elevations, $\hat{z}$, with composile DEM, $z_{\mathrm{a}}$

\begin{tabular}{crrr}
\hline $\begin{array}{c}\text { Double-difference } \\
\text { interferogram }\end{array}$ & $\mu_{\hat{z}-z_{\mathrm{a}}}$ & $\sigma_{\bar{z}-z_{\mathrm{A}}}$ & \multicolumn{1}{c}{$\begin{array}{c}\sigma_{\bar{z}-\mathrm{za}} z \\
\geq 1400 \mathrm{~m}\end{array}$} \\
& $\mathrm{~m}$ & $\mathrm{~m}$ & $\mathrm{~m}$ \\
\hline$I_{2}-I_{1}$ & 0.66 & 7.57 & 6.24 \\
$2 \times I_{2}-I_{3}$ & 0.72 & 4.41 & 3.54 \\
$2 \times I_{2}-I_{4}$ & -3.02 & 11.24 & 10.46 \\
$2 \times I_{1}-I_{3}$ & 2.79 & 6.84 & 4.10 \\
$I_{4}-I_{3}$ & 1.40 & 9.79 & 8.69 \\
$2 \times I_{1}-I_{4}$ & -1.41 & 5.94 & 3.96 \\
& & &
\end{tabular}


phase-unwrapping.

The differences between the composite and individual DEMs indicate that there may still be some unanticipated phase error in the interferograms. Shaded surfaces (Fig. 5) are useful for observing differences among DEMs. Random phase error due to speckle appears as a high-frequency pattern because it varies independently from pixel to pixel. This error tends to be larger for images with shorter baselines. For example, the shaded surface in Figure $5 \mathrm{~b}$ is more textured than the surface of the DEM shown in Figure $5 \mathrm{a}$, which corresponds to a much longer baseline. Errors that show up as horizontal lines can be seen in the DEMs illustrated in Figure 5 as well as in the DEMs that are not shown. These errors are the result of minor phase discontinuities in the complex SAR images. Neither of these effects is large enough to explain the large standard deviations.

The DEM shown in Figure $5 b$ has structures that appear as long ridges or valleys and extend nearly horizontally most of the way across the frame. In contrast, the DEM in Figure 5a is relatively free of such structures. Many of the other individual DEMs (not shown) have

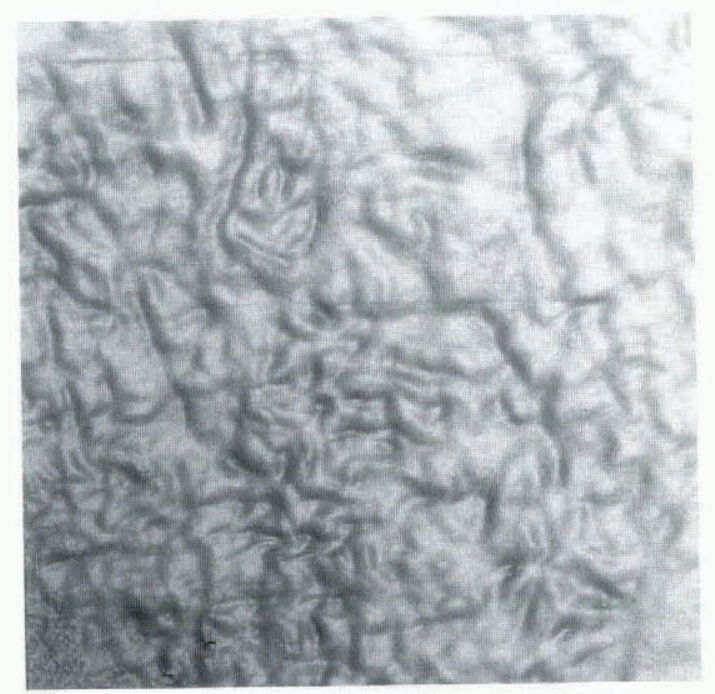

a

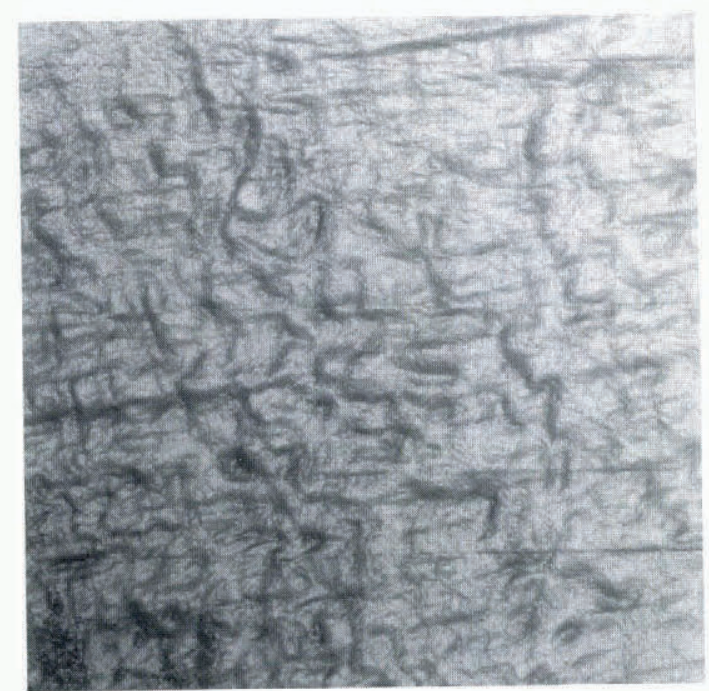

b

Fig. 5. Shaded surfaces of individual DEMs generated from interferograms (a) $2 \times I_{1}-I_{3}$ and (b) $2 \times I_{2}-I_{4}$. features with shapes and orientation of the same kind as the features visible in Figure 5b. They are not topographic features, because they differ from DEM to DEM. These "streaks" tend to average out in the composite DEM so that little of this type of structure can be seen in Figure 4.

The phase fluctuations that cause streak error are estimated by subtracting an interferogram simulated using the composite DEM from an individual double-difference interferogram. Phase error estimated in this way for $I_{2}-I_{1}$ and $2 \times I_{2}-I_{4}$ are displayed modulo- $2 \pi$ in Figure 6 . Although the estimates of phase error in Figure 6 correspond to interferograms that have similar baseline lengths, the magnitudes of the streaks in each interferogram

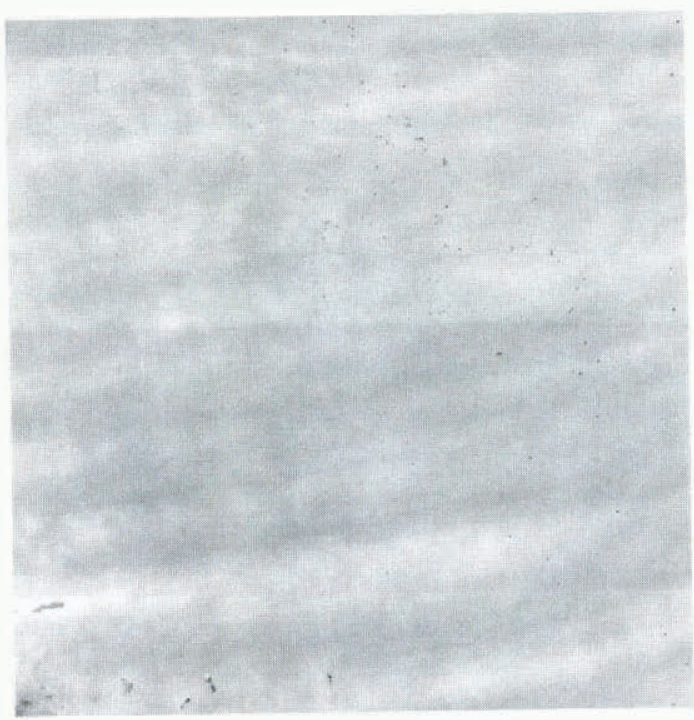

a

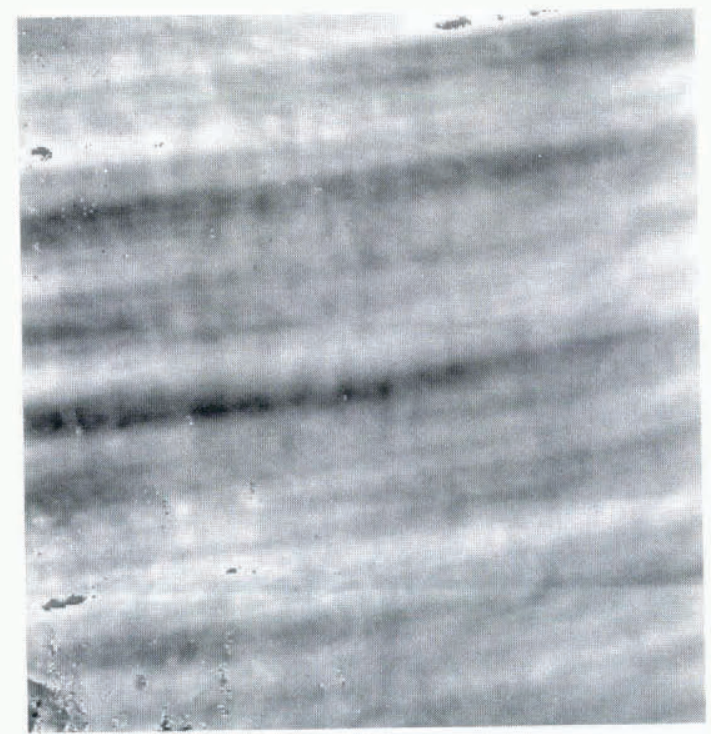

b

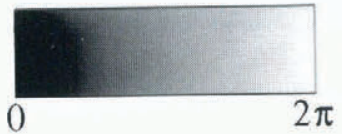

Fig. 6. Residual phase after subtracting the effect of topography from phase-corrected interferograms for (a) $I_{2}-I_{1}\left(B_{11} \approx-106 \mathrm{~m}\right)$, and (b) $2 \times I_{2}-I_{4}\left(B_{\mathrm{n}} \approx\right.$ $118 \mathrm{~m})$. 
differ greatly. Observations of similar estimates from several other interferograms also indicate that streak intensity is independent of baseline length. The sensitivity of a DEM to streak and other phase errors is inversely proportional to baseline length. Thus, the best DEMs are achieved for interferograms having both low streak error and long baselines. Because the widths of the streaks are of similar scale to the topographic undulations, it is difficult to estimate and remove this error by means of filtering.

There are similar streaks in the correlation images for the interferograms. Jezek and Rignot (1994) have also observed streaks with similar orientation in correlation images from a nearby ERS-1 swath. They have shown that the streaks are related to high-frequency variations of the azimuth registration. The underlying cause of this streak "noise" is still unknown.

\section{Comparison with laser-altimeter data}

The NASA Airborne Oceanographic Lidar (AOL) has made several flights over the Greenland ice sheet. This laser altimeter performs overlapping conical scans about $150 \mathrm{~m}$ in diameter. Eighty elevation measurements are made per scan. AOL measurements of ice-sheet elevations are repeatable to within $10 \mathrm{~cm}$ (Krabill and others, 1995 , in press). Accuracy is on the order of a few centimeters, so comparison with $\mathrm{AOL}$ data provides a good estimate of the accuracy of interferometric DEMs.

An AOL flight line from 27 July 1993 crosses the upper righthand corner of frame 2169. Figure 3 shows the location of frame 2169 and the AOL flight line. The portion of the flight line that intersects the SAR frame is $76 \mathrm{~km}$ long. The AOL data are subsampled along the center of the scan to obtain measurements with $80 \mathrm{~m}$ spacing, which is approximately the pixel spacing of the interferometric DEMs. To allow comparison with interferometric DEMs, the elevation, latitude and longitude of each point along the profile are converted to the ground-range coordinates of the SAR frame. It might have been possible to obtain more accurate baseline estimates by using tie points from the AOL data. This was not attempted, however, because the goal is to establish the accuracy of DEMs created using the radaraltimetry tie points alone.

The mean, $\mu_{\hat{z}-z_{A O L}}$, and standard deviation, $\sigma_{\hat{z}-z_{A O L}}$, of the difference between the AOL elevations, $z_{\mathrm{AOL}}$, and the interferometrically derived elevations, $\hat{z}$, are reported in Table 4 . The values of $\mu_{\hat{z}-z_{A O L}}$ vary from about 0 to $5 \mathrm{~m}$. The mean difference between the AOL profile and the profile extracted from the KMS DEM is $3.43 \mathrm{~m}$. Thus, the mean difference of $3.81 \mathrm{~m}$ for the composite DEM is mostly attributable to differences between the AOL profile and KMS DEM. Streak noise probably contributes to some of the variability in the mean differences directly, and also indirectly through an increase in baseline estimation error.

Standard deviations for the individual DEMs are all in rough agreement with the results from the comparison of composite and individual DEMs (Table 3). Only two of the individual DEMs differ from the AOL data by significantly more than $5 \mathrm{~m}$. The relative accuracy of the composite DEM is $2.56 \mathrm{~m}$, which is almost equal to that of the best individual DEM.

Relative differences in elevation for the AOL data, the composite DEM and the best individual DEM are
Table 4. Comparison of interferometrically measured elevations, $\hat{z}$, with $A O L$ elevations, $z_{\mathrm{AOL}}$

\begin{tabular}{ccr}
\hline$D E M$ & $\mu_{\hat{z}-z_{\mathrm{AOL}}}$ & $\sigma_{\hat{z}-z_{\mathrm{AOL}} .}$ \\
& $\mathrm{m}$ & $\mathrm{m}$ \\
\hline$I_{2}-I_{1}$ & 4.95 & 5.12 \\
$2 \times I_{2}-I_{3}$ & 4.42 & 4.71 \\
$2 \times I_{2}-I_{4}$ & 0.05 & 10.07 \\
$2 \times I_{1}-I_{3}$ & 5.20 & 2.55 \\
$I_{4}-I_{3}$ & 5.86 & 10.01 \\
$2 \times I_{1}-I_{4}$ & 2.49 & 4.41 \\
Composite DEM & 3.81 & 2.56 \\
\hline
\end{tabular}

identified by comparing the profiles after subtraction of the average elevation from each profile (Fig. 7). DEM error is largest (about $5 \mathrm{~m}$ ) for the first $10 \mathrm{~km}$ of the profile, but stays below about $2.5 \mathrm{~m}$ for the rest of the profile. This comparison demonstrates that it is possible to generate ice-sheet DEMs with relative error of about $2.5 \mathrm{~m}$. Elimination of streak noise and better tie points (i.e. AOL data) should further reduce elevation error.

Error from random-phase noise due to speckle varies nearly independently from pixel to pixel. Therefore, the fairly smooth interferometric profiles indicate that this type of phase noise is a relatively minor source of error. The AOL profile is smooth i.e., there is little vertical variation greater than $1 \mathrm{~m}$ ) on the scale of the 4 (range) by 20 (azimuth) multilook pixel size, so considerably more multilook averaging could be applied to further reduce what little phase noise there is from speckle.

There may be considerable penetration at C-band (i.e. up to $25 \mathrm{~m}$ for dry snow; Mätzler, 1987), so that the measured surface differs from the actual surface. Any bias created by penetration to a uniform depth will be removed during the baseline estimation procedure, which tends to fix the average height to that of the tie points. Spatially varying depth of penetration could

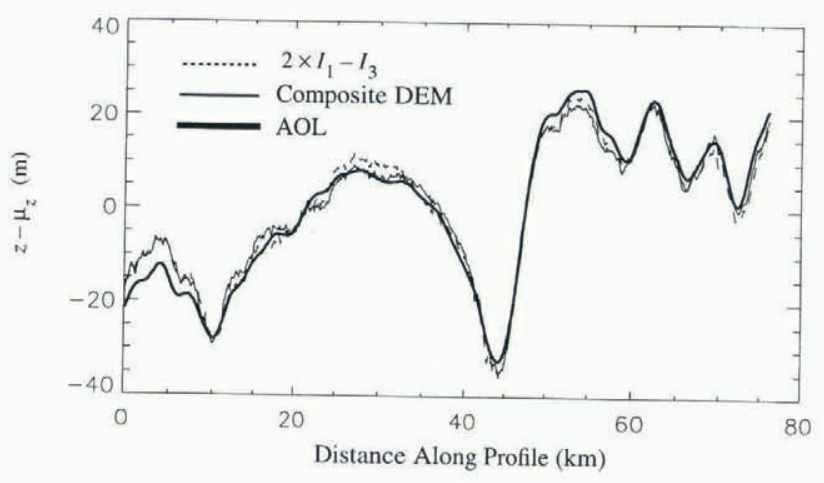

Fig. 7. Comparison of AOL profiles with the composite DEM and with the DEM computed using $2 \times I_{1}-I_{3}$. To illustrate relative differences the mean elevation is subtracted from each profile. The profile runs from. NW to $S E$ and corresponds to that part of the $A O L$ flight line illustrated that intersects frame 2169 (Fig. 3). 

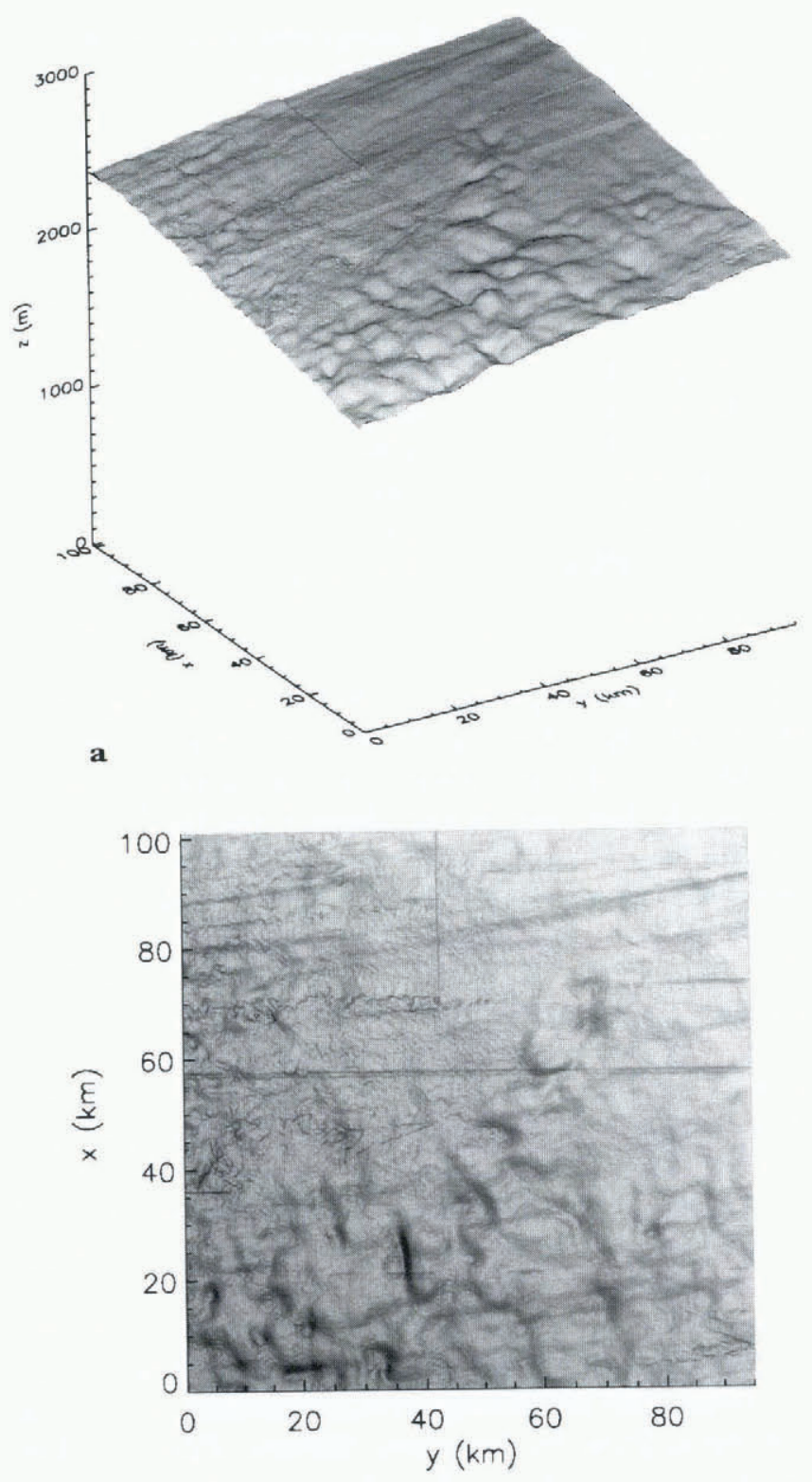

b

Fig. 8. Shaded surface of the composite DEM for frame 2151. The vantage-point is (a) from the side and (b) from above. The light source is directed from above, along the $z$ axis.

cause relative differences between the measured topography and the actual surface topography. Comparison with AOL data indicates that these differences are less than $2.5 \mathrm{~m}$ in the area we have examined, which is in the percolation zone. We have not done a comparison for other snow facies, where penetration is likely quite different. The effect of penetration on interferometric DEM accuracy is a topic that needs further study.

\section{DEMS FOR OTHER FRAMES}

Interferometric DEMs are also derived for the frames adjacent to 2169: frames 2151 and 2187. The orbits used are the same as those given in Table 1. The baselines are similar to those listed in Tables 1 and 2. Several of the interferograms for frame 2151 have many streaks and generally low correlation. Satisfactory DEMs can only be created for $I_{2}-I_{1}, 2 \times I_{1}-I_{3}$, and $2 \times I_{1}-I_{4}$. Figure 8 shows the composite DEM for frame 2151 formed using these three DEMs. Because even the interferometric pairs for these DEMs are not strongly correlated, topographic errors in the DEMs for this frame are greater than for the DEMs from frame 2169. Some of this additional error is seen in Figure 8 as streaks and minor discontinuities.

The DEM for frame 2151 shows a sharp transition from a gently undulating surface, like that seen in frame 2169 , to much smoother topography at higher elevations. Because the scale of dynamically supported topography is determined by ice thickness, velocity and bedrock topography (Paterson, 1981; Johannesson, 1992), this transition most likely reflects the effects of thicker ice and lower surface slopes toward the interior of the ice sheet.

Figure 9 shows the composite DEM for frame 2187. A large region corresponding to an outlet glacier is almost
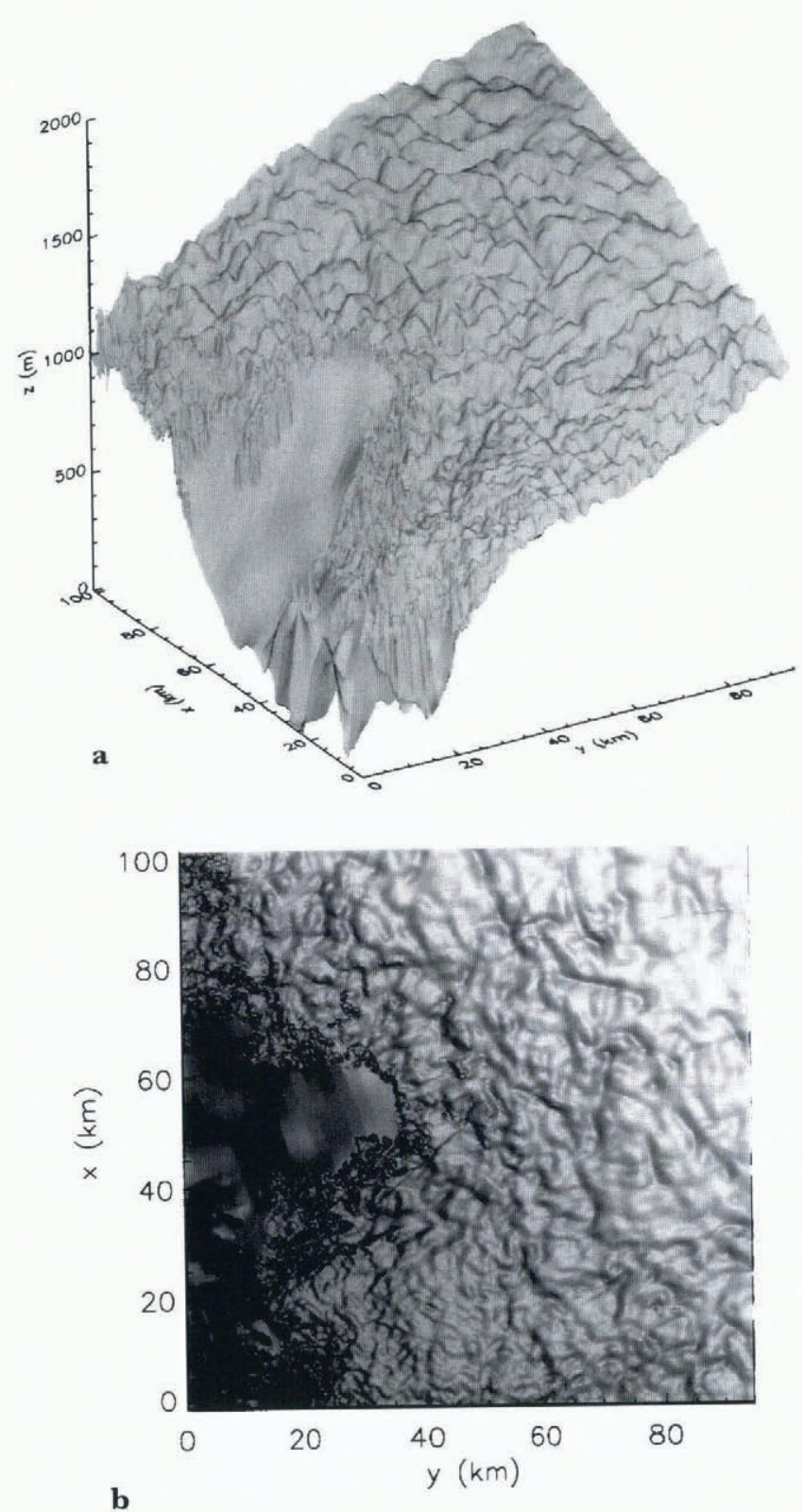

Fig. 9. Shaded surface of the composite DEM for frame 2187. The vantage-point is (a) from the side and (b) from above. The light source is directed from above, along the $z$ axis. 
completely decorrelated. This region could not be unwrapped for any of the interferograms. The missing elevations in this area have been replaced with elevations from the KMS DEM. The interferograms for this frame have more streak errors than those for frame 2169. As a result, DEM accuracy is likely to be less than that for frame 2169. Nevertheless, a lack of visible streaks on the righthand side of Figure 9 indicates that a reasonable level of accuracy (i.e. a few meters) may have been achieved.

The DEMs in earlier figures are presented in the ground-range coordinates of the SAR frame. For most applications it is desirable to represent the DEM in some other geographic coordinate system, such as the polar stereographic coordinates of the SSM/I grid. This coordinate transformation can be made using a geocoding procedure (Joughin, 1995). Because the interferometric equations yield both elevation and ground range, terrain-correction is accomplished automatically $\mathrm{Li}$ and Goldstein, 1990). Transforming the DEMs into a common coordinate system makes it is possible to combine and compare them with other elevation data sets. For example, Figure 10 shows the shaded surface of the interferometric DEMs for frames 2151, 2169 and 2187 combined with a section of the KMS DEM. The increase in topographic detail offered by interferometry is striking.

\section{TOPOGRAPHY AND PHASE DUE TO ICE MOTION}

Motion causes complex fringe patterns in ice-sheet interferograms. For example, the interferograms in Figure 2a and b have many closed-phase contours due to motion that resemble bull's-eyes. An understanding of the complexity seen in these fringe patterns is needed before estimates of velocity can be made. In particular, it is important to determine the relative contributions of vertical and horizontal motion to the interferometric phase. Joughin and others (1995) used a simple model to demonstrate that bull's-eye patterns are caused by vertical displacement as ice flows over bumps in the surface topography. Their model indicates that, over length scales of less than a few ice thicknesses, phase variation is dominated by vertical velocity. This is because the steep $\left(23^{\circ}\right)$ incidence angle of the ERS-1 SAR makes phase more sensitive to longitudinal gradients in vertical velocity than to longitudinal gradients in horizontal velocity, by a factor of roughly 2.4. Over longer length scales, where the magnitude of the slope variation is not as great, phase variation is dominated by horizontal displacement. In this section we confirm this analysis by comparing phase variation due to motion with the surface topography of our interferometrically derived DEM.

We begin by using the DEM from frame 2169 to simulate and remove the direct effect of topography (i.e. $\left.\phi_{\text {topography }}\right)$ from the interferogram formed with images from orbits 3089 and 3132 . The phase, $\phi_{\text {displacement, is }}$ unwrapped and then high-pass filtered to remove variation with wavelengths longer than $7 \mathrm{~km}$, which corresponds to at least three ice thicknesses. Topography and high-pass filtered $\phi_{\text {displacement }}$ for a $96 \mathrm{~km}$ by $86 \mathrm{~km}$ subsection of frame 2169 are compared in Figure 11. The DEM is shown as a shaded surface with a view from above. The light source is directed downslope to illuminate slopes in the approximate direction of flow. Uphill sides of bumps are illuminated, while downhill sides appear dark. The contours of the high-pass filtered phase are plotted over the shaded surface. A variable contour interval is used. For magnitudes less than or equal to $2 \pi$, red and blue contours with an interval of $\pi / 2$ are used. For larger magnitudes green and cyan correspond to contours with a $2 \pi$ interval. Red and cyan are used for contours with negative values, and green and blue for those with positive values.

Positive contours correspond strongly with downhill sides of undulations, while negative contours correspond with uphill sides. This is exactly what would be expected

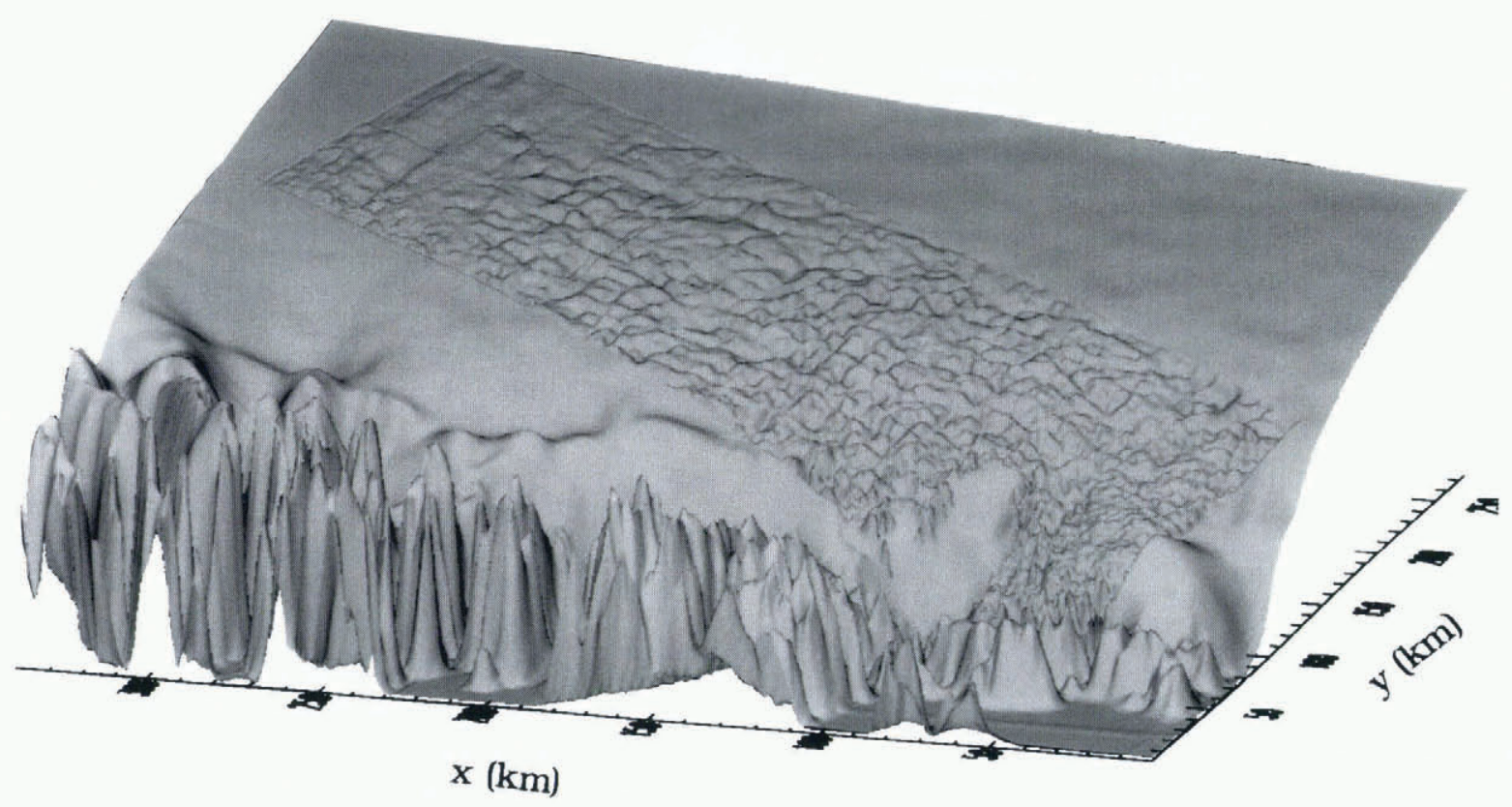

Fig. 10. Shaded surface of the combination of interferometric DEMs for frames 2151, 2169 and 2187 and parl of the KMS DEM. The light source is directed from above, along the zaxis. 

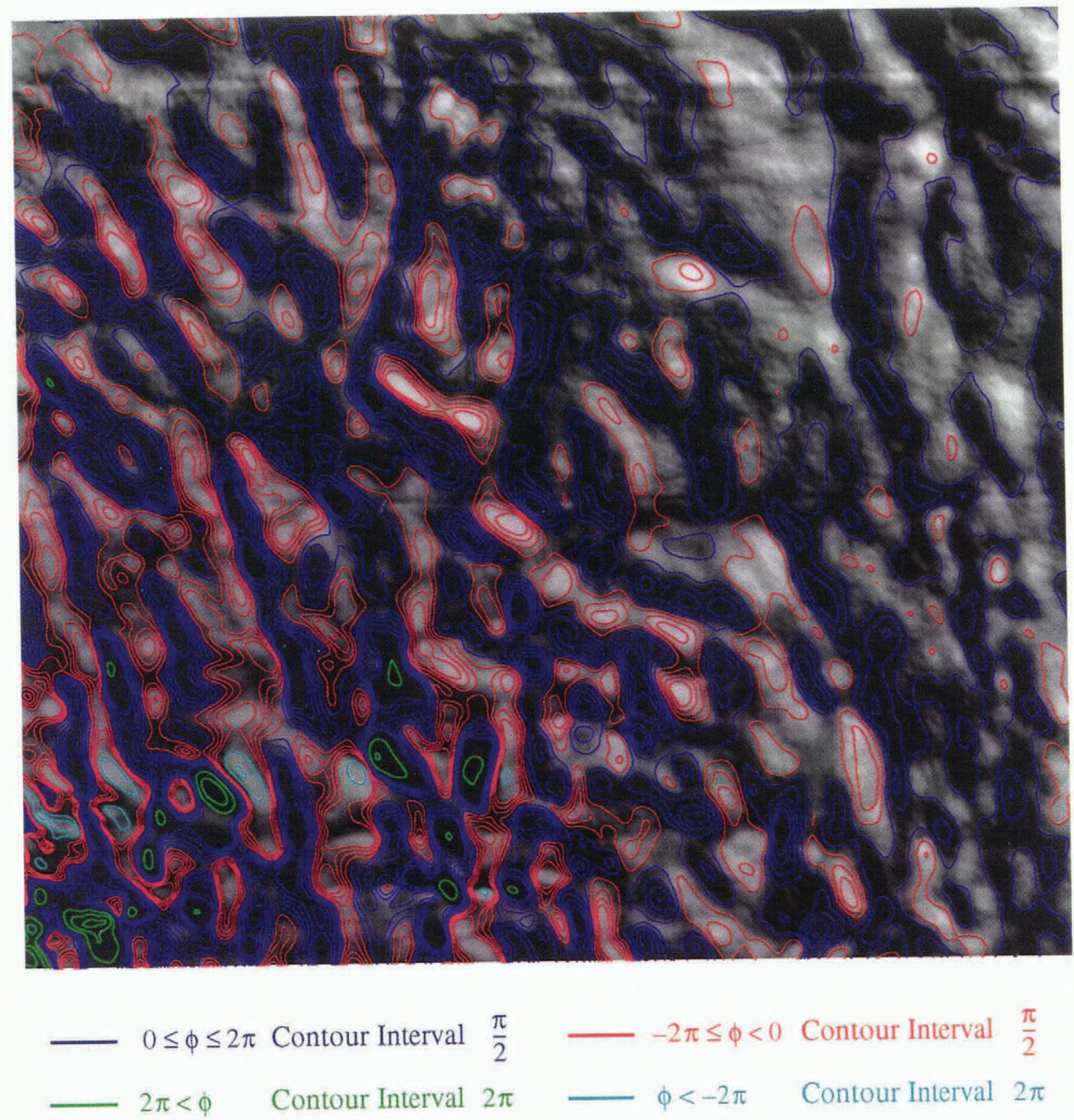

Fig. 11. Contours of the high-pass filter phase plotted over the shaded surface of the DEM for a subsection of frame 2169. The light source is directed downslope from top right to bottom left.

if the short-scale phase variation were dominated by the effects of vertical displacement. On the uphill side of a bump, deviation from the average slope is positive, so that vertical motion is upwards, causing the phase to decrease. On the downhill side, downward ice motion results in positive-phase variation. The changes from positive to negative phases (from blue to red contours in Figure 11) generally follow the areas of dark-to-light transition. These transitions correspond to peaks and valleys where the slope magnitudes are at a minimum. This close agreement between the high-pass filtered phase and surface slope is strong support for the conclusion that for ice sheets, small-scale phase variation (i.e. bull's-eyes) is dominated by the effect of vertical rather than horizontal motion.

\section{GONCLUSIONS}

Ice-sheet DEMs can be generated successfully from interferograms that have been differenced to cancel motion. Comparison of a laser-altimeter-measured topographic profile with data from the interferometrically derived DEMs yields relative errors as low as $2.55 \mathrm{~m}$. Absolute elevation accuracy of about $4 \mathrm{~m}$ is achieved using radar-altimetry tie points. Thus, interferometric DEMs can provide elevation data with the accuracy needed for many glaciological investigations (a goal of $5 \mathrm{~m}$ is suggested by Thomas and others (1985)). It is likely that these accuracies can be improved with better processing and more accurate tie-point information.

Most of the topographic error in the interferometrically derived DEMs is caused by variations in the registration offsets in the azimuth direction, which show up as streaks in the correlation and phase images. The cause of these streaks is unknown. Because streak error is a major limitation to DEM accuracy, further research is needed to determine its cause and to find ways to eliminate it. If streak error can be eliminated, it may be possible to achieve accuracy below $2 \mathrm{~m}$. This is because the small surface slopes of the ice sheets allow extensive 
multilook averaging to reduce random-phase noise due to speckle, which is often the limiting factor to interferometric DEM accuracy. The relatively small slopes of the ice sheets also mean that large slope-related errors (i.e. layover regions) that affect interferometric accuracy are not a factor.

There are unexplained low-frequency errors in the double-difference interferograms. These errors can be removed using a radar-altimetry-derived DEM. This is not an ideal solution, however, because any longwavelength errors in the altimetry-derived DEM are propagated to the interferometrically derived DEM. More research is needed to determine the cause of this type of error. Then it may be possible to eliminate the error without the aid of an altimetry-derived DEM.

Our results are important to the consideration of future interferometers. The accuracies reported here are for DEMs created from double-difference, repeat-pass interferograms. These interferograms are subject to far more noise than single-difference, single-pass interferograms. Significantly better topographic accuracy could be achieved if a single-pass, spaceborne interferometer, such as TOPSAT (Zebker and others, 1994b), were built.

We have demonstrated that double-difference interferograms can be used to cancel motion even when the interferograms are acquired nearly 2 years apart. This is significant because it means that interferograms acquired during the ERS-1/ERS-2 tandem mission can be used to create ice-sheet DEMs. Those interferograms will have temporal baselines of only $1 \mathrm{~d}$, so they should yield better results than the 3 and $6 \mathrm{~d}$ interferograms used in this work.

Interferometry has been shown capable of providing much more detailed topographic data than radar altimetry. Nevertheless, interferometry is not a substitute for altimetry. Interferometry depends on tie points for absolute accuracy. In contrast, radar altimetry measures absolute elevation, but provides limited topographic detail. Interferometric DEMs may allow improvements in the accuracy of radar-altimeter estimates of elevation (personal communication from R.A. Bindschadler, 1994). Undulations in the ice-sheet topography cause errors in radar-altimetry data. Interferometric measurements of the relative topography, which include these undulations, potentially could be used to remove surfaceinduced errors in radar altimetric measurements.

While other studies Goldstein and others, 1993; Joughin and others, 1995; Rignot and others, 1995) have used satellite-radar interferometry to examine icesheet motion, we have applied this technique for measuring the detailed topography of ice sheets. We have compared our high-resolution estimates of topography with motion-only interferograms to confirm an earlier analysis (Joughin and others, 1995) that shortscale phase variation is dominated by the effect of vertical displacement. It is important to note that a powerful aspect of interferometry is its ability to collect coincident motion and topography data. Combined high-resolution motion and topographic data should aid in the understanding of ice-sheet flow dynamics and help glaciologists to test and refine their models (personal communication from H. Gudmundsson, 1994).

\section{ACKNOWLEDGEMENTS}

I. Joughin and D. Winebrenner carried out this research under NASA grant NAGW-1256. M. Fahnestock was supported by NMTPE grant 5121-PP-0129. R. Kwok performed this work at the Jet Propulsion Laboratory, California Institute of Technology, under contract with NASA. W. Krabill conducted this research at NASA Goddard Space Flight Center, Wallops Flight Facility.

We thank S. Ekholm of the National Survey and Cadastre, Copenhagen, Denmark, for providing us with the DEM. We also thank the anonymous reviewers for their many helpful comments.

\section{REFERENCES}

Bindschadler, R. A. 1984. Jakobshavns glacier drainage basin: a balance assessment. J. Geophys. Res., 89 (C2), 2066-2072.

Bindschadler, R.A., ed. 1991. West Antarctic Ice Sheel Initiative. Volume 1. (NASA Conference Publication 3115.

Bindschadler, R. A. and P. L. Vornberger. 1994. Detailed elevation map of Ice Stream C, Antarctica, using satellite imagery and airborne radar. Ann. Glaciol., 20, 327-335.

Gabriel, A. K., R. M. Goldstein and H. A. Zebker. 1989. Mapping small elevation changes over large areas: differential radar interferometry. J. Geophys. Res., 94 B7), 9183-9191.

Goldstein, R. M., H. A. Zebker and C. L. Werner. 1988. Satellite radar interferometry: two-dimensional phase unwrapping. Radio Sci., 23 (4), $713-720$.

Goldstein, R. M.. H. Engelhardt, B. Kamb and R. M. Frolich. 1993. Satellite radar interferometry for monitoring ice sheet motion: application to an Antarctic ice stream. Science, 262 (5139), 1525-1530.

Jezek, K. and E. Rignot. 1994. Katabatic wind processes on the Greenland ice sheet. EOS, 75 44), Supplement, 212.

Johannesson, T. 1992. Landscape of temperate ice caps. Ph.D. thesis, University of Washington.

Joughin, I. R. 1995. Estimation of ice-sheet topography and motion using interferometric synthetic aperture radar. $\mathrm{Ph} . \mathrm{D}$. thesis, University of Washington.

Joughin, I. R., D. P. Winebrenner and M. A. Fahnestock. 1995. Observations of ice-sheet motion in Greenland using satellite radar interferometry. Geophys. Res. Lell., 22 (5), 571-574.

Krabill, W. B., R.H. Thomas, C.F. Martin, R. N. Swift and E. B. Frederick. 1995. Accuracy of airborne laser altimetry over the Greenland ice sheet. Int. 7. Remote Sensing, 16 7), 1211-1222.

Krabill, W. B., R. H. Thomas, K. Jezek, K. Kuivinen and S. Manizade. In press. Greenland ice sheet thickness changes measured by laser altimetry. Geophys. Res. Letl.

Kwok, R. and M. Fahnestock. 1996. Ice-sheet motion and topography from radar interferometry. IEEE Trans. Geosci. Remote Sensing. 34 (1)

Li, F. K. and R.M. Goldstein. 1990. Studies of multi-baseline spaceborne interferometric synthetic aperture radars. IEEE Trans. Geosci. Remole Sensing, GE-28 (1), 8897.

Mattar, K. E., A. L. Gray, M. W. A. vander Kooij and P.J. FarrisManning. 1994. Airborne interferometric SAR results from mountainous and glacial terrain. In Stein, T. 1., ed. IGARSS '94. Surface and Atmospheric Remote Sensing: Technologies, Data Analysis and Interpretation. Vol. IV. California Institute of Technology, Pasadena, California USA, August 8-12, 1994. Pisacataway, NJ, Institute of Electrical and Electronics Engineers, 2388-2396.

Mătzler, C. 1987. Applications of the interaction of microwaves with the natural snow cover. Remote Sensing Rez.. 2 2) 259387.

Paterson, W. S. B. 1981. The physics of glaciers. Second edition. Oxford, etc., Pergamon Press.

Rignot, E., K. C. Jezek and H. G. Sohn. 1995. Ice flow dynamics of the Greenland ice sheet from SAR interferometry. Geophys. Res, Lell., 22 (5), 575-578.

Rodriguez, E. and J.M. Martin. 1992. Theory and design of interferometric synthetic aperture radars. IEE Proc., Ser. F, 139 (2), $147-159$.

Smith, A. M., C. Wilson and P. J. Meadows. 1994. The EODC SAR processor. Int. J. Remote Sensing, 15 4), 785-801. 
Solaas, G. A. 1994. ERS-I SAR interferometric baseline algorithm verification. Version 1.1. Frascati, Italy, ESA/ESRIN. (No. ES-TN-DPE-OMGS02.

Thomas, R. H. and 8 others. 1985. Satellite remole sensing for ice sheet research. Washington, DC, National Aeronautics and Space Administration. (NASA Tech. Memo. 86233.

Vander Veen, C.J. 1991. State of balance of the cryosphere. Rev. Geophys., 29 (3), 433-455.

Zebker, H.A. and R.M. Goldstein. 1986. Topographic mapping from interferometric synthetic aperture radar observations. 7. Geophys.
Res., 91 B.5), $4993-4999$

Zebker, H. A. and 8 others. 1992. The TOPSAR interferometric radar topographic mapping instrument. IEEE Trans. Geosci. Remote Sensing, GE-30 (5), $933-940$.

Zebker, H.A., C. L. Werner, P.A. Rosen and S. Hensley. 1994a. Accuracy of topographic maps derived from ERS-1 interferometry. IEEE Trans. Geosci. Remote Sensing, GE-32 4), 823-836.

Zebker, H.A., T. G. Farr, R.P. Salazar and T.H. Dixon. 1994b. Mapping the world's topography using radar interferometry: the TOPSAT mission. Proc. IEEE, 82 (12), 1774-1786.

MS received 3 July 1995 and accepted in revised form 22 September 1995 